\title{
Disease-specific Graded Prognostic Assessment
}

National Cancer Institute

\section{Source}

National Cancer Institute. Disease-specific Graded Prognostic Assessment. NCI

Thesaurus. Code C140883.

A 4-tiered score that stratifies patients with common primary tumours metastasizing to the brain (malignant melanoma, lung, breast, kidney and gastrointestinal cancers) into subgroups with different prognoses. It takes into account that brain metastases from different primary tumor sites respond differently to radiation therapy. 